\title{
Bernhard Weimer
}

\section{Die mozambiquanische Außenpolitik 1975-1982}

\section{Merkmale, Probleme, Dynamik}

Aktuelle Materialien zur internationalen Politik, Band II.

Herausgegeben von der Stiftung Wissenschaft und Politik, Ebenhausen. Nomos Verlagsgesellschaft, Baden-Baden, 1983, 213 Seiten, DM 22,-.

Es ist das Schicksal sozialwissenschaftlicher Analysen, bereits bei Erscheinen teilweise überholt zu sein. Die Gründe dafür liegen auf der Hand. Zum einen ist der sozio-politische Wandel im internationalen System extrem hoch, zum anderen gelingt es uns nicht, sämtliche Determinanten dieses Wandels exakt herauszuarbeiten. Aussagekräftiges Material ist häufig nicht vorhanden, veraltet oder es bleibt der Forschung verschlossen, da die Neigung der Herrschenden, Einblick in die Innenausstattung der Macht zu gewähren, nicht sonderlich ausgeprägt ist.

B. Weimers Studie ist da keine Ausnahme, zumal er sich auf ein ebenso notwendiges wie anspruchsvolles Unternehmen eingelassen hat. Monographien über das lusophone Afrika sind aus historischen, sprachlichen und - nicht zuletzt - forschungspraktischen Gründen rar. Trotz dieser Schwierigkeiten will Weimer (zu Recht) mit seiner Arbeit zur Außenpolitik der Volksrepublik Mozambique Informationsdefizite beseitigen und verbreitete Vorurteile ("Mozambique als Handlanger des expansionistischen Weltkommunismus") abbauen.

Die Studie ist aus regionalistischer Perspektive geschrieben, ohne globale Zusammenhänge außer Acht zu lassen. Sie basiert auf einer Position, die sich wohl am besten mit 'kritischer Sympathie zur Frelimo-Regierung' umschreiben läßt. Daß Weimer beides explizit macht, dient der Objektivität seiner Argumentation. (Leider versäumen globalistische und dezidiert antikommunistische Arbeiten über das lusophone Afrika nicht selten, die normative Basis ihrer Aussagen transparent zu machen.)

Methodologisch handelt es sich überwiegend um eine - nicht unkritische - Beschreibung der wichtigsten Ziele, Dimensionen und Handlungsobjekte der mozambiquanischen Außenpolitik, die mit eigenen analytischen Kategorien angereichert ist.

Mozambique ist - so Weimers Ausgangsthesen - von Südafrika wirtschaftlich-sstrukturelle, von den sozialistischen Staaten, insbesondere von der UdSSR und der DDR, militärisch-’konjunkturell abhängig. Je nach Intensität und Dauer der südafrikanischen Destabilisierungsaktionen - entweder durch direkte militärische Intervention der südafrikanischen Streitkräfte oder durch indirekte Sabotageaktivitäten der Renamo - vergrößert oder verringert sich die "konjunkturelle" Abhängigkeit Mozambiques von der Militärhilfe des Ostblocks. Folglich ist es das Ziel der mozambiquanischen Außenpolitik, "strukturelle" ökonomische Abhängigkeiten der Volksrepublik abzubauen und die mozambiquanische Revolution vor innerer und äußerer Stabilisierung durch Südafrika zu schützen. Obwohl daher deutliche sicherheitspolitische, ökonomische und auch ideologische Interessenkonvergenzen mit der "entwickelten sozialistischen Staatengemein- 
schaft" bestehen, bemüht sich Mozambique um eine eigenständige, pragmatische Interessenpolitik.

Sie kommt etwa gegenüber den sozialistischen Staaten in folgenden Bereichen zum Ausdruck:

(1)

Mozambique hat der Roten Flotte Stützpunkte verweigert.

(2)

Im Befreiungskampf Zimbabwes unterstützte die Frelimo-Regierung die Zanu R. Mugabes, während die UdSSR diplomatisch, politisch wie militärisch J. Nkomos Zapu Hilfe gewährte.

(3)

Mozambique versucht, seine militärischen Beziehungen zu diversifizieren. Es hat nicht nur regionale Militär- und Sicherheitsabkommen (mit Zimbabwe und Tanzania) abgeschlossen, das Land arbeitet auch sicherheitspolitisch-militärisch mit Portugal (der ehemaligen Kolonialmacht, gegen die die Frelimo gekämpft hat) und Frankreich zusammen.

(4)

Wirtschaftlich ist die Frelimo-Regierung an einer Zusammenarbeit vor allem mit der EG interessiert. Nach Anerkennung der - besser einer - Berlinklausel nimmt Mozambique inzwischen an den Lomé III-Verhandlungen teil.

(5)

In jüngster Zeit ist außenpolitisch und wirtschaftlich zudem eine Annäherung an die Volksrepublik China und an die USA erkennbar.

Obwohl die Frelimo-Regierung den Marxismus-Leninismus als universelle Gesellschaftstheorie übernommen hat, sich in ihrer anti-imperialistischen Rhetorik, zuweilen auch in der außenpolitischen Praxis, an die UdSSR anlehnt (Mozambique stimmte in der Afghanistan- und der Kamputschea-Frage mit der Sowjetunion überein), ist Weimars Fazit überzeugend: Die Volksrepublik Mozambique sei keineswegs als sowjetischer client state einzuschätzen. Es liege daher im langfristigen Interesse auch der bundesrepublikanischen Außenpolitik, die Kooperation - im weitesten Sinne - mit der Volksrepublik zu suchen. Das heißt, der Bonner Rhetorik 'Respektierung der nationalen Souveränität', 'eigener wirtschaftlicher und kultureller Leitbilder', 'Blockfreiheitı etc. auch entsprechende Taten folgen zu lassen.

Wo Licht ist, ist bekanntlich auch Schatten:

- Die Ausgangsthese "strukturelle" Abhängigkeit von Südafrika, "konjunkturelle" Abhängigkeit von der UdSSR hätte es, (vom nicht unproblematischen begrifflichen Vokabular abgesehen) verdient, noch umfassender und systematischer belegt zu werden. Merkwürdigerweise rangieren beide Staaten, auf der - auch vom Autor selbst als methodologisch problematisch betrachteten - Liste der wichtigsten Länder für die mozambiquanische Außenpolitik an nachrangiger Stelle.

Angesichts der unzureichenden Datenlage mußte Weimer sich stattdessen damit 
begnügen, die verschiedenen Handlungsobjekte der mozambiquanischen Außenpolitik (westliche Länder, sozialistische Länder, schwarzafrikanische Länder) vorzustellen. Ob allerdings die Beziehungen zu 34 Staaten (multilaterale Beziehungen ausgeschlossen) auf knapp einhundert Seiten hinreichend analysiert werden können, sei dahingestellt. Möglicherweise wäre hier 'weniger mehr' gewesen, das heißt, eine bewußte und begründete Analyse der Beziehungen zu Schwerpunktländern wie Südafrika und der UdSSR.

- Die Binnen-Determinanten der mozambiquanischen Außenpolitik (wirtschaftliche Entwicklung, ethnische und regionale Konflikte, ideologische Differenzen innerhalb der Frelimo, Pressure Groups etc.) werden nicht oder nur am Rande behandelt, obwohl sie bei der Formulierung dieser Politik eine nicht unerhebliche Rolle gespielt haben.

Diese - dem Autor angesichts der dürftigen Datenlage kaum anzulastenden - Defizite haben beachtliche Konsequenzen. Südafrika ist es - auch infolge der Hungerkrisen in Mozambique - inzwischen gelungen, das Nachbarland an den Verhandlungstisch zu bomben. Mit dem Vertrag von Nkomati hat Pretoria unter dem Beifall der Westmächte im südlichen Afrika den ersten Schritt zur Etablierung eines Cordon Sanitaire wirtschaftlich abhängiger und politisch-'pazifizierter` Randstaaten gemacht. Der NkomatiVertrag - praktisch ein wechselseitiges Anti-Subversionsabkommen - macht den südafrikanischen Rassenkonflikt vertraglich zu einem internen Menschenrechtsproblem der Republik. Er verpflichtet Mozambique zu wirtschaftlich-technischer und sicherheitspolitischer Zusammenarbeit. Mit Beitritt der Volksrepublik Mozambique zum Internationalen Währungsfonds und zur Weltbank zeichnet sich zudem ein Rapprochement an 'kapitalistischer Finanz- und Entwicklungsorganisationen ab.

Nicht Dissoziation sondern Kooperation, nicht Internationalisierung, sondern Renationalisierung des südafrikanischen Rassenkonflikts scheinen jetzt die neuen Formeln mozambiquanischer Außenpolitik zu sein - alles Entwicklungen, die aus Weimers Analyse kaum abzuschätzen sind.

Er befindet sich dabei in guter Gesellschaft. Da unsere Prognosen auf einer Konstanz bestimmter Daten basieren müssen (sonst wären sie nicht möglich), haben sie einen konservativen 'bias', das heißt, sie extrapolieren Bekanntes.

Alles in allem also ein informativer und detailreicher 'tour d'horizon', der wertvolle Anstöße zu vertieften (Spezial-) Analysen geben kann. Das ist nicht eben wenig.

Gerald Braun 